\title{
A Note on Topological Entropy of Continuous Self-Maps
}

\author{
Zabidin Salleh \\ School of Informatics and Applied Mathematics, Universiti Malaysia Terengganu, 21030 Kuala Terengganu, Terengganu, Malaysia.
}

Received: December 24, 2014 / Accepted: January 22, 2015 / Published: March 25, 2015.

\begin{abstract}
Topological entropy can be an indicator of complicated behavior in dynamical systems. It is first introduce by Adler, Konheim and McAndrew by using open covers in 1965. After that it is still an active research by many researchers to produce more properties and applications up to nowadays. The purpose of this paper is to review and explain most important concepts and results of topological entropies of continuous self-maps for dynamical systems on compact and non-compact topological and metric spaces. We give proofs for some of its elementary properties of the topological entropy. Slight modification on Adler's topological entropy is also presented.
\end{abstract}

Keywords: Dynamical system, topological entropy, continuous maps, compact space, metric space.

\section{Introduction}

Let $X$ be a Hausdorff topological space and $f: X \rightarrow X$ be a continuous self-map on $X$. The pair $(X, f)$ is called a dynamical system. If $n \in \mathbb{N}$, then $f^{n}=f \circ f^{n-1}, f^{1}=f$ and $f^{0}$ is the identity map on $X$. For $x \in X$, the sequence $\left\{f^{n}(x): n \in \mathbb{N}\right\}$ is called the orbit of $x$. If $f$ is a homeomorphism, the full orbit of $x$ is the sequence $\left\{f^{n}(x): n \in \mathbb{Z}\right\}$.

Topological entropy can be an indicator of complicated (chaotic) behavior in dynamical systems. Whether the topological entropy of a dynamical system is positive or not is of primary significance, due to the fact that positive topological entropy implies that one can assert that the system is chaotic. It is hard, as remarked by [9], to get a good idea of what topological entropy means directly from various definitions of topological entropy. Thus it is enough to know that topological entropy of a dynamical system is a measure of complexity of dynamic behavior of the

system, and it can be seen as a quantitative measurement of how chaotic of a dynamical system. Generally speaking, the larger the topological entropy of a system is the more complicated the dynamics of

Corresponding author: Zabidin Salleh, Ph.D., research field: topological dynamics. E-mail: zabidin@umt.edu.my. this system would be.

This paper is organized as follow. In section 2, we review the earlier concept of topological entropy introduced by Adler et al. [1] as an invariant of topological conjugacy and also as an analogue of measure theoretic entropy. This topological entropy only holds on a compact topological space. In section 3 , we review and discuss another definition of topological entropy introduced by Bowen [2] using separating and spanning sets on metric space. This definition holds not only on compact space but also on non-compact space. Finally in the last section, slight modification of Adler's topological entropy has been done by changing the symbol of refinement.

\section{Topological Entropy for Maps on Compact Spaces}

The original definition of topological entropy begins in 1965 by Adler et al. [1]. They introduce the topological entropy of a continuous self-map $f: X \rightarrow X$ on a compact space $X$. So in this section, we always let $X$ be a nonempty compact topological space, and $f: X \rightarrow X$ a continuous self-map.

Definition 2.1. For any open cover $\mathcal{U}$ of $X$, let $N(\mathcal{U})$ denote the number of sets in a subcover of minimal cardinality. A subcover of a cover is minimal 
if no other subcover contains fewer members. We call $H(\mathcal{U})=\log N(\mathcal{U})$ the entropy of $\mathcal{U}$.

Since $X$ is compact and $\mathcal{U}$ is an open cover, there always exists a finite subcover. Then we have $H(\mathcal{U})=\log \min \{\operatorname{card}(\mathcal{V}): \mathcal{V}$ a finite subcover of $\mathcal{U}\}$.

Definition 2.2. For any two covers $\mathcal{U}, \mathcal{v}$ of $X$, $\mathcal{U} \vee \mathcal{V} \equiv\{A \cap B: A \in \mathcal{U}, B \in \mathcal{V}\}$ defines their join. Similarly we can define the join $\vee_{k=1}^{n} \mathcal{U}_{k}=$ $\left\{\bigcap_{k=1}^{n} A_{k}: A_{k} \in \mathcal{U}_{k}\right\}$ of any finite collection of open covers of $X$.

Let $f: X \rightarrow X$ be a continuous mapping on a compact space $X$. If $\mathcal{U}$ is an open cover of $X$, then from continuity, the family $f^{-1}(\mathcal{U})=\left\{f^{-1}(U): U \in\right.$ $\mathcal{U}$ is again an open cover of $X$. Sometimes we will write $f^{-1} \mathcal{U}$ instead of $f^{-1}(\mathcal{U})$ if there is no chance for confusion. Similarly for $f \mathcal{U}$.

The following proposition state the properties of $N(\mathcal{U})$ and $H(\mathcal{U})$.

Proposition 2.1. Let $\mathcal{U}, \mathcal{v}$ be two open covers of a compact space $X$. Then

(i) $N(\mathcal{U} \vee \mathcal{V}) \leq N(\mathcal{U}) \cdot N(\mathcal{V})$ and $H(\mathcal{U} \vee \mathcal{V}) \leq$ $H(\mathcal{U})+H(\mathcal{V})$

(ii) $f^{-1}(\mathcal{U} \vee \mathcal{V})=f^{-1}(\mathcal{U}) \vee f^{-1}(\mathcal{V})$;

(iii) $\quad N(\mathcal{U}) \geq N\left(f^{-1}(\mathcal{U})\right)$ and $H(\mathcal{U}) \geq H\left(f^{-1}(\mathcal{U})\right)$. If $f$ is onto, we have equality.

(iv) $N(f \mathcal{U}) \geq N(\mathcal{U})$ and $H(f \mathcal{U}) \geq H(\mathcal{U})$. If $f$ is homeomorphism, we have equality.

Proof. (i) Let $\left\{A_{1}, \ldots, A_{N(u)}\right\}$ and $\left\{B_{1}, \ldots, B_{N(\mathcal{V})}\right\}$ be subcovers of $U$ and $\mathcal{V}$ of minimal cardinality, respectively. Then

$$
\left\{A_{i} \cap B_{j}: i=1, \ldots, N(\mathcal{U}), j=1, \ldots, N(\mathcal{V})\right\}
$$

is a subcover of $\mathcal{U} \vee \mathcal{V}$ (possibly not minimal) of cardinality $N(\mathcal{U}) \cdot N(\mathcal{V})$. Consequently $N(\mathcal{U} \vee \mathcal{V}) \leq$ $N(\mathcal{U}) \cdot N(\mathcal{V})$.

(ii) By the property of inverse image,

$$
\begin{aligned}
f^{-1}(\mathcal{U} \vee \mathcal{V})=\{ & \left.f^{-1}(A \cap B): A \in \mathcal{U}, B \in \mathcal{V}\right\} \\
& =\left\{f^{-1}(A) \cap f^{-1}(B): A \in \mathcal{U}, B \in \mathcal{V}\right\} \\
& =f^{-1}(\mathcal{U}) \vee f^{-1}(\mathcal{V}) .
\end{aligned}
$$

(iii) Let $\left\{A_{1}, \ldots, A_{N(U)}\right\}$ be a subcover of $\mathcal{U}$ of minimal cardinality. Since $\left\{f^{-1}\left(A_{1}\right), \ldots, f^{-1}\left(A_{N}(u)\right)\right\}$ is also a subcover of $f^{-1} \mathcal{U}$, possibly not minimal, we have $N\left(f^{-1} \mathcal{U}\right) \leq N(\mathcal{U})$. If $f$ is onto and $\left\{f^{-1}\left(A_{1}\right), \ldots, f^{-1}\left(A_{N\left(f^{-1} u\right)}\right)\right\}$ is a subcover of $f^{-1} \mathcal{U}$ of minimal cardinality, then also $\left\{A_{1}, \ldots, A_{N\left(f^{-1} u\right)}\right\}$ is a subcover of $\mathcal{U}$. Thus we have, $N\left(f^{-1} \mathcal{U}\right) \geq N(\mathcal{U})$.

(iv) Let $\left\{f A_{1}, \ldots, f A_{N(f u)}\right\}$ be a subcover of $f \mathcal{U}$ of minimal cardinality. Since $\left\{f^{-1} f\left(A_{1}\right), \ldots, f^{-1} f\left(A_{N(f u)}\right)\right\}$ is also a subcover of $f^{-1} f \mathcal{U}$, possibly not minimal, we have $N\left(f^{-1} f \mathcal{U}\right) \leq$ $N(f \mathcal{U})$. Since $\mathcal{U} \subseteq f^{-1} f \mathcal{U}, N(\mathcal{U}) \leq N\left(f^{-1} f \mathcal{U}\right)$ and therefore $N(\mathcal{U}) \leq N(f \mathcal{U})$. If $f$ is homeomorphism and $\left\{f^{-1} f\left(A_{1}\right), \ldots, f^{-1} f\left(A_{N\left(f^{-1} f u\right)}\right)\right\}$ is a subcover of $f^{-1} f \mathcal{U}$ of minimal cardinality, then also $\left\{f A_{1}, \ldots, f A_{N\left(f^{-1} f u\right)}\right\}$ is a subcover of $f \mathcal{U}$. Thus we have, $\quad N\left(f^{-1} f \mathcal{U}\right) \geq N(f \mathcal{U})$. Since $f$ is homeomorphism, $\mathcal{U}=f^{-1} f \mathcal{U}$, then $N(\mathcal{U})=$ $N\left(f^{-1} f \mathcal{U}\right) \geq N(f \mathcal{U})$.

Remark.(i) The operation $\vee$ is obviously commutative and associative.

(ii) $f^{-1}\left(\mathrm{~V}_{k=0}^{n-1} \mathcal{U}_{k}\right)=\bigvee_{k=0}^{n-1} f^{-1}\left(\mathcal{u}_{k}\right)$.

Lemma 2.1. For every open cover $U$ of $X$, the following

$$
\begin{gathered}
\lim _{n \rightarrow \infty} \frac{1}{n} H\left(\vee_{k=0}^{n-1} f^{-k}(\mathcal{U})\right) \\
=\lim _{n \rightarrow \infty} H\left(\mathcal{U} \vee f^{-1}(\mathcal{U}) \vee \cdots \vee f^{-n+1}(\mathcal{U})\right)
\end{gathered}
$$

exists and is a nonnegative real number.

Proof. Consider the sequence $c_{n}=H\left(\mathrm{~V}_{k=0}^{n-1} f^{-k}(\mathcal{U})\right), n \in \mathbb{N}$. Since $X$ is nonempty, every open cover of $X$ contains at least one set, and therefore $c_{n} \geq 0$ for all $n \in \mathbb{N}$. Proposition 2.1 implies that $\left(c_{n}: n \in \mathbb{N}\right)$ is subadditive, i.e., $c_{m+n} \leq c_{m}+c_{n}$ for all $m, n \in \mathbb{N}$ :

$$
\begin{aligned}
& c_{m+n}=H\left(\mathrm{~V}_{k=0}^{m+n-1} f^{-k}(\mathcal{U})\right) \\
& =H\left(\mathcal{U} \vee f^{-1} \mathcal{U} \vee \cdots \vee f^{-m-n+1} \mathcal{U}\right) \\
& =H\left(\mathcal{U} \vee f^{-1} \mathcal{U} \vee \cdots \vee f^{-m+1} \mathcal{U} \vee f^{-m} \mathcal{U} \vee \cdots\right. \\
& \left.\vee f^{-m-n+1} \mathcal{u}\right) \\
& =H\left(\mathcal{U} \vee f^{-1} \mathcal{U} \vee \cdots \vee f^{-m+1} \mathcal{U}\right. \\
& \left.\vee f^{-m}\left(\mathcal{U} \vee \cdots \vee f^{-n+1} \mathcal{u}\right)\right) \\
& \leq H\left(\mathcal{U} \vee f^{-1} \mathcal{U} \vee \cdots \vee f^{-m+1} \mathcal{U}\right) \\
& +H\left(f^{-m}\left(\mathcal{U} \vee \cdots \vee f^{-n+1} \mathcal{u}\right)\right)
\end{aligned}
$$




$$
\begin{aligned}
& \leq H\left(\mathcal{U} \vee f^{-1} \mathcal{U} \vee \cdots \vee f^{-m+1} \mathcal{U}\right) \\
& +H\left(\mathcal{U} \vee \cdots \vee f^{-n+1} \mathcal{u}\right) \\
& =c_{m}+c_{n} \text {. }
\end{aligned}
$$

The fourth equality follows from Proposition 2.1(ii); the next inequality from Proposition 2.1 (i); and the final inequality from Proposition 2.1 (iii). From the subadditivityof $\left(c_{n}\right)$, clearly it is a decreasing sequence. It follows by an elementary result from real analysis that the $\lim _{n \rightarrow \infty} c_{n}$ exists and equals $\inf _{n \in \mathbb{N}} c_{n}$. Thus the $\lim _{n \rightarrow \infty} \frac{c_{n}}{n}$ exists and is a nonnegative real number.

Definition 2.3. The topological entropy of $f$ with respect to an open cover $\mathcal{U}$ is defined by

$$
h(f, \mathcal{U})=\lim _{n \rightarrow \infty} \frac{1}{n} H\left(\bigvee_{k=0}^{n-1} f^{-k}(\mathcal{U})\right),
$$

and the topological entropy of $f$ is defined as $h(f)=\sup _{u}\{h(f, \mathcal{U}): \mathcal{U}$ is an open cover of $X\}$, where the supremum is taken over all open covers $\mathcal{U}$ of $X$. Obviously, $h(f) \in[0, \infty]$.

Lemma 2.2. Let $\mathcal{U}$ be an open cover of $X$. Then $h(f, \mathcal{U}) \leq H(\mathcal{U})$.

Proof. By Definition 2.3, Proposition 2.1 (i) and (iii),

$$
\begin{gathered}
h(f, \mathcal{U})=\lim _{n \rightarrow \infty} \frac{1}{n} H\left(\mathcal{U} \vee f^{-1} \mathcal{U} \vee \cdots \vee f^{-n+1} \mathcal{U}\right) \\
\leq \lim _{n \rightarrow \infty} \frac{1}{n}\left[H(\mathcal{U})+H\left(f^{-1} \mathcal{U}\right)+\cdots+H\left(f^{-n+1} \mathcal{U}\right)\right] \\
\leq \lim _{n \rightarrow \infty} \frac{1}{n}[H(\mathcal{U})+H(\mathcal{U})+\cdots+H(\mathcal{U})] \\
=\lim _{n \rightarrow \infty} \frac{1}{n}[n H(\mathcal{U})]=H(\mathcal{U}) .
\end{gathered}
$$

Definition 2.4. A cover $\mathcal{V}$ is said to be a refinement of a cover $u$, denoted as $u \prec v$, if every element of $\mathcal{V}$ is a subset of some element of $\mathcal{U}$. In other words, $U \prec \mathcal{V}$ if $A \in \mathcal{V}$, there exists a $B \in \mathcal{U}$ such that $A \subseteq B$.

Lemma 2.3. Let $\mathcal{U}, \mathcal{v}, \mathcal{U}^{\prime}$ and $\mathcal{V}^{\prime}$ be open covers of $X$. Then the following statements hold:

(i) $u<U^{\prime}, v \prec v^{\prime} \Rightarrow U \vee v \prec \mathcal{U}^{\prime} \vee v^{\prime}$.

(ii) $\mathcal{U} \prec \mathcal{V} \Rightarrow N(\mathcal{U}) \leq N(\mathcal{V})$ and $H(\mathcal{U}) \leq H(\mathcal{V})$. (iii) $U \prec \mathcal{V} \Rightarrow N(\mathcal{U} \vee \mathcal{V})=N(\mathcal{V})$ and $H(\mathcal{U} \vee \mathcal{V})=H(\mathcal{V})$

(iv) $U \prec \mathcal{V} \Rightarrow f^{-1}(\mathcal{U}) \prec f^{-1}(\mathcal{V})$

(v) $u \prec v \Rightarrow h(f, \mathcal{U}) \leq h(f, \mathcal{v})$.

Proof. (i) Consider $A^{\prime} \cap{ }^{\prime} B \in \mathcal{U}^{\prime} \vee \mathcal{V}^{\prime}$ where $A^{\prime} \in \mathcal{U}^{\prime}$ and $B^{\prime} \in \mathcal{V}^{\prime}$. By hypothesis there exists $A \in \mathcal{U}$ and $B \in \mathcal{V}$ such that $A^{\prime} \subseteq A$ and $B^{\prime} \subseteq B$. Thus $A^{\prime} \cap B^{\prime} \subseteq A \cap B$ where $A \cap B \in \mathcal{U} \vee \mathcal{V}$.

(ii) Let $\left\{B_{1}, \ldots, B_{N(\mathcal{V})}\right\}$ be a subcover of $\mathcal{V}$ of minimal cardinality. Since $u<v$, there exists a subcover $\left\{A_{1}, \ldots, A_{N(v)}\right\}$ of $U$ such that $A_{i} \subseteq B_{i}$ for $i=1, \ldots, N(\mathcal{V})$. Therefore $N(\mathcal{U}) \leq N(\mathcal{V})$.

(iii) Obviously by (i), $\mathcal{V}=\{X\} \vee \mathcal{V} \prec \mathcal{U} \vee \mathcal{V}$ so that $N(\mathcal{V}) \leq N(\mathcal{U} \vee \mathcal{V})$ by (ii). On the other hand, $\mathcal{U} \prec \mathcal{v}$ and $\mathcal{v} \prec \mathcal{v}$ implies $u \vee v \prec \mathcal{v} \vee \mathcal{v} \prec \mathcal{V}$. Thus $N(\mathcal{U} \vee \mathcal{V}) \leq N(\mathcal{V})$.

(iv) Let $A \in f^{-1}(\mathcal{V})$, then $f(A) \in \mathcal{V}$. Since $\mathcal{U} \prec \mathcal{V}$, there exists $B \in \mathcal{U}$ such that $f(A) \subseteq B$. This implies that $A \subseteq f^{-1}(f(A)) \subseteq f^{-1}(B) \in f^{-1}(\mathcal{U})$. Thus $f^{-1}(\mathcal{U}) \prec f^{-1}(\mathcal{V})$.

(v) By part (i) and (iv), it follows that $u \vee f^{-1} \mathcal{U} \vee$ $\cdots \vee f^{-n+1} u \prec \mathcal{v} \vee f^{-1} \mathcal{v} \vee \cdots \vee f^{-n+1} \mathcal{V} \quad$ for all $n \in \mathbb{N}$. Therefore by (ii),

$$
\begin{aligned}
H\left(\mathcal{U} \vee f^{-1} \mathcal{U} \vee\right. & \left.\cdots \vee f^{-n+1} \mathcal{U}\right) \\
& \leq H\left(\mathcal{V} \vee f^{-1} \mathcal{V} \vee \cdots \vee f^{-n+1} \mathcal{V}\right)
\end{aligned}
$$

This yields the claim.

Theorem 2.1. If $\phi: X \rightarrow Y$ is a continuous surjection and $g: Y \rightarrow Y$ a continuous map such that $\phi \circ f=g \circ \phi$, then $h(g) \leq h(f)$. If $\phi$ is a homeomorphism, then $h(f)=h(g)$.

Proof. If $\mathcal{U}$ is an open cover of $Y$, then $\phi^{-1}(\mathcal{U})$ is an open cover of $X$ and

$$
\begin{aligned}
& h\left(f, \phi^{-1}(\mathcal{U})\right)=\lim _{n \rightarrow \infty} \frac{1}{n} H\left(\mathrm{~V}_{k=0}^{n-1} f^{-k}\left(\phi^{-1} \mathcal{U}\right)\right) \\
& =\lim _{n \rightarrow \infty} \frac{1}{n} H\left(\bigvee_{k=0}^{n-1} \phi^{-1}\left(g^{-k} \mathcal{U}\right)\right) \\
& =\lim _{n \rightarrow \infty} \frac{1}{n} H\left(\phi^{-1} \bigvee_{k=0}^{n-1} g^{-k} \mathcal{U}\right) \\
& =\lim _{n \rightarrow \infty} \frac{1}{n} H\left(\bigvee_{k=0}^{n-1} g^{-k}(\mathcal{U})\right) \\
& =h(g, \mathcal{U})
\end{aligned}
$$


by Proposition 2.1 (iii). It follows that

$$
\begin{aligned}
& h(f)=\sup _{\mathcal{V}}\{h(f, \mathcal{V}): \mathcal{V} \text { is an open cover of } X\} \\
\geq & \sup _{\phi^{-1} \mathcal{U}}\left\{h\left(f, \phi^{-1} \mathcal{U}\right): \phi^{-1} \mathcal{U} \text { is an open cover of } X\right\} \\
= & \sup _{\mathcal{U}}\{h(g, \mathcal{U}): \mathcal{U} \text { is an open cover of } Y\} \\
= & h(g) .
\end{aligned}
$$

If $\phi$ is a homeomorphism and if $\mathcal{U}$ is an open cover of $X$, then $\phi(\mathcal{U})=\left(\phi^{-1}\right)^{-1}(\mathcal{U})$ is an open cover of $Y$ and we have $\phi^{-1} \circ g=f \circ \phi^{-1}$. So

$$
\begin{aligned}
& h(g, \phi \mathcal{U})=h\left(g,\left(\phi^{-1}\right)^{-1}(\mathcal{U})\right) \\
= & \lim _{n \rightarrow \infty} \frac{1}{n} H\left(\mathrm{~V}_{k=0}^{n-1} g^{-k}\left(\left(\phi^{-1}\right)^{-1} \mathcal{U}\right)\right) \\
= & \lim _{n \rightarrow \infty} \frac{1}{n} H\left(\left(\phi^{-1}\right)^{-1} \bigvee_{k=0}^{n-1} f^{-k}(\mathcal{U})\right) \\
= & \lim _{n \rightarrow \infty} \frac{1}{n} H\left(\mathrm{~V}_{k=0}^{n-1} f^{-k}(\mathcal{U})\right)=h(f, \mathcal{U})
\end{aligned}
$$

by Proposition 2.1 (iii). It follows that

$$
h(g)=\sup _{\mathcal{V}}\{h(g, \mathcal{V}): \mathcal{V} \text { is an open cover of } Y\}
$$

$\geq \sup _{\phi \mathcal{U}}\{h(g, \phi \mathcal{U}): \phi \mathcal{U}$ is an open cover of $Y\}$

$=\sup _{u}\{h(f, \mathcal{U}): \mathcal{U}$ is an open cover of $X\}$

$$
=h(f) \text {. }
$$

Corollary 2.1. Let $f: X \rightarrow X$ be a continuous mapping and let $\sim$ be an equivalence relation on $X$ such that $f(x) \sim f(y)$ whenever $x \sim y$. Let $\tilde{f}: X / \sim \rightarrow X / \sim$ be the mapping defined by $\tilde{f} \circ \pi=$ $\pi \circ f$ where $\pi$ is the projection of $X$ onto $X / \sim$. Then $h(\tilde{f}) \leq h(f)$.

Proof. The projection $\pi: X \rightarrow X / \sim$ is a continuous surjection with $\tilde{f} \circ \pi=\pi \circ f$. The result follows by Theorem 2.1

Theorem 2.2. Topologically conjugate maps have the same topological entropy, $h(f)=h\left(g \circ f \circ g^{-1}\right)$ where $f: X \rightarrow X$ is a continuous map and $g: X \rightarrow Y$ is a homeomorphism.

Proof. Let $\mathcal{U}$ be an open cover of $X$. Since $g$ is a homeomorphism, $g \mathcal{U}$ is an open cover of $Y$. Hence

$$
\begin{aligned}
& h\left(g \circ f \circ g^{-1}, g \mathcal{U}\right) \\
& =\lim _{n \rightarrow \infty} \frac{1}{n} H\left(\vee_{i=0}^{n-1}\left(g \circ f \circ g^{-1}\right)^{-i}(g \mathcal{U})\right) \\
& =\lim _{n \rightarrow \infty} \frac{1}{n} H\left(g \mathcal{U} \vee g \circ f^{-1} \circ g^{-1} g \mathcal{U} \vee \cdots \vee g \circ f^{-n+1}\right. \\
& \left.\quad \circ g^{-1} g \mathcal{U}\right) \\
& =\lim _{n \rightarrow \infty} \frac{1}{n} H\left(\mathcal{U} \vee f^{-1} \mathcal{U} \vee \cdots \vee f^{-n+1} \mathcal{u}\right)=h(f, \mathcal{u}) .
\end{aligned}
$$

The third equality follows from Proposition 2.1. Hence $h(f)=h\left(g \circ f \circ g^{-1}\right)$

Theorem 2.3. (i) For the topological entropy of iterates the formula $h\left(f^{k}\right)=k \cdot h(f)$ holds for all $k \in \mathbb{N}$.

(ii) If $f$ is a homeomorphism, then $h(f)=$ $h\left(f^{-1}\right)$ and therefore $h\left(f^{k}\right)=|k| h(f)$ for all $k \in \mathbb{Z}$.

Proof. (i) For any open cover $U$ of $X$ we have

$$
\begin{aligned}
& h\left(f^{k}\right)=\sup _{u}\left\{h\left(f^{k}, \mathcal{U}\right): \mathcal{U} \text { is an open cover of } X\right\} \\
& \geq h\left(f^{k}, \vee_{i=0}^{k-1} f^{-i}(\mathcal{U})\right) \\
& =\lim _{n \rightarrow \infty} \frac{1}{n} H\left(\mathrm{\vee}_{i=0}^{n-1}\left(f^{k}\right)^{-i}\left(\vee_{i=0}^{k-1} f^{-i}(\mathcal{U})\right)\right) \\
& =\lim _{n \rightarrow \infty} \frac{1}{n} H\left(\mathcal{U} \vee f^{-1} \mathcal{U} \vee \cdots \vee f^{-n k+1} \mathcal{U}\right) \\
& =k \lim _{n \rightarrow \infty} \frac{1}{n k} H\left(\vee_{i=0}^{n k-1} f^{-i}(\mathcal{U})\right) \\
& =k h(f, \mathcal{U}) .
\end{aligned}
$$

Thus $h\left(f^{k}\right) \geq k h(f)$. On the other hand, since for any open cover $\mathcal{U}$,

$$
\vee_{i=0}^{n-1}\left(f^{k}\right)^{-i} \mathcal{U} \prec \bigvee_{i=0}^{n k-1} f^{-i} \mathcal{U},
$$

we have

$$
\begin{gathered}
h(f, \mathcal{U})=\lim _{n \rightarrow \infty} \frac{1}{n k} H\left(\mathrm{~V}_{i=0}^{n k-1} f^{-i}(\mathcal{U})\right) \\
\geq \lim _{n \rightarrow \infty} \frac{1}{n k} H\left(\mathrm{~V}_{i=0}^{n-1}\left(f^{k}\right)^{-i} \mathcal{u}\right) \\
=\frac{1}{k} \lim _{n \rightarrow \infty} \frac{1}{n} H\left(\mathrm{~V}_{i=0}^{n-1}\left(f^{k}\right)^{-i} \mathcal{U}\right) \\
=\frac{1}{k} h\left(f^{k}, \mathcal{u}\right)
\end{gathered}
$$

by Lemma 2.3(ii). Thus $k h(f) \geq h\left(f^{k}\right)$.

(ii) If $f$ is a homeomorphism and $u$ any open 
cover of $X$, then

$$
\begin{gathered}
h(f, \mathcal{U})=\lim _{n \rightarrow \infty} \frac{1}{n} H\left(\vee_{k=0}^{n-1} f^{-k}(\mathcal{U})\right) \\
=\lim _{n \rightarrow \infty} \frac{1}{n} H\left(f^{n-1}\left(\vee_{k=0}^{n-1} f^{-k}(\mathcal{U})\right)\right) \\
=\lim _{n \rightarrow \infty} \frac{1}{n} H\left(\mathcal{U} \vee f \mathcal{U} \vee \cdots \vee f^{n-1} \mathcal{U}\right) \\
=\lim _{n \rightarrow \infty} \frac{1}{n} H\left(\mathcal{U} \vee\left(f^{-1}\right)^{-1} \mathcal{U} \vee \cdots \vee\left(f^{-1}\right)^{-n+1} \mathcal{U}\right) \\
=h\left(f^{-1}, \mathcal{U}\right) .
\end{gathered}
$$

Thus $h(f)=h\left(f^{-1}\right)$. If $k<0$, then $h\left(f^{k}\right)=h\left(\left(f^{-1}\right)^{-k}\right)=-k h\left(f^{-1}\right)=|k| h(f)$.

This yields the statement.

\section{Topological Entropy for Maps on Metric Spaces}

Bowen [2] defines the topological entropy $h(f)$ of a uniformly continuous map $f: X \rightarrow X$ on an arbitrary metric space $(X, d)$ (not necessarily compact) via $(n, \varepsilon)$-separated and $(n, \varepsilon)$-spanning sets. In general, this quantity depends on the metric $d$. But for $X$ being compact it coincides with the topological entropy as defined by Adler et al. [1].

Definition 3.1. Let $(X, d)$ be a metric space and $f: X \rightarrow X$ a uniformly continuous map (abbreviated: $f \in \operatorname{UC}(X, d))$. For each $n \in \mathbb{N}, d_{n, f}(x, y)=$ $\max _{0 \leq i \leq n-1} d\left(f^{i}(x), f^{i}(y)\right)$ defines a metric on $X$, topologically equivalent to $d$. A set $E \subseteq X$ is $(n, \varepsilon)$-separated if for all $x, y \in E$ with $x \neq y$ it holds that $d_{n, f}(x, y)>\varepsilon$. A set $F \subseteq X$ is $(n, \varepsilon)$-spans another set $K \subseteq X$ (with respect to $f$ ) if for every $x \in K$ there is a $y \in F$ with $d_{n, f}(x, y) \leq$ $\varepsilon$.

Definition 3.1. For a compact set $K \subseteq X$, let $r_{n}(\varepsilon, K)$ be the smallest cardinality of a set which $(n, \varepsilon)$-spans $K$, and let $s_{n}(\varepsilon, K)$ be the largest cardinality of an $(n, \varepsilon)$-separated set contained in $K$. We write $r_{n}(\varepsilon, K, f)$ or $s_{n}(\varepsilon, K, f)$ if we wish to stress the dependence on $f$. Define

$$
r(\varepsilon, K, f)=\limsup _{n \rightarrow \infty} \frac{1}{n} \log r_{n}(\varepsilon, K),
$$

$$
s(\varepsilon, K, f)=\limsup _{n \rightarrow \infty} \frac{1}{n} \log s_{n}(\varepsilon, K) .
$$

\section{Lemma 3.1.}

(i) $r_{n}(\varepsilon, K) \leq s_{n}(\varepsilon, K) \leq r_{n}\left(\frac{1}{2} \varepsilon, K\right)<\infty$.

(ii) For $\varepsilon_{1}<\varepsilon_{2}, r\left(\varepsilon_{1}, K, f\right) \geq r\left(\varepsilon_{2}, K, f\right)$ and $s\left(\varepsilon_{1}, K, f\right) \geq s\left(\varepsilon_{2}, K, f\right)$.

By the Lemma 3.1, the following definition makes sense.

Definition 3.2. For $f \in \mathrm{UC}(X, d)$ and $K \subseteq X$ compact, $\quad$ set $\quad h_{d}(f, K)=\lim _{\varepsilon \rightarrow 0} r(\varepsilon, K, f)=$ $\lim _{\varepsilon \rightarrow 0} s(\varepsilon, K, f)$, and one defines the topological entropy of $f$ is $h_{d}(f)=\sup \left\{h_{d}(f, K): K \subseteq\right.$ $X$ compact.

In Definition 3.2, we stress the dependence on the metric $d$ used. Metrics $d$ and $d^{\prime}$ on $X$ are uniformly equivalent if $i d_{X}:(X, d) \rightarrow\left(X, d^{\prime}\right)$ and $i d_{X}:\left(X, d^{\prime}\right) \rightarrow(X, d)$ are both uniformly continuous maps of metric spaces. In this case $f \in \operatorname{UC}(X, d)$ if and only if $f \in \operatorname{UC}\left(X, d^{\prime}\right)$. Bowen [2] proves the following properties of $h_{d}(f)$.

Theorem 3.1. (i) If $d_{1}$ and $d_{2}$ are uniformly equivalent metrics on $X$, then $h_{d_{1}}(f)=h_{d_{2}}(f)$.

(ii) For iterates it holds that $h_{d}\left(f^{m}\right)=m \cdot h_{d}(f)$ for all $f \in \operatorname{UC}(X, d)$ and $m \in \mathbb{N}$.

Proof. (i) Given $\varepsilon_{1}>0$, choose $\varepsilon_{2}>0$ such that $d_{2}(x, y)<\varepsilon_{2}$ implies $d_{1}(x, y)<\varepsilon_{1}$ and choose $\varepsilon_{3}>0$ such that $d_{1}(x, y)<\varepsilon_{3}$ implies $d_{2}(x, y)<\varepsilon_{2}$. Let $K \subseteq X$ be compact, then an $\left(n, \varepsilon_{2}\right)$-spanning set for $K$ with respect to $d_{2}$ is an $\left(n, \varepsilon_{1}\right)$-spanning set for $K$ with respect to $d_{1}$. Hence $r_{n}\left(\varepsilon_{1}, K, d_{1}\right) \leq$ $r_{n}\left(\varepsilon_{2}, K, d_{2}\right) . \quad$ Similarly $\quad r_{n}\left(\varepsilon_{2}, K, d_{2}\right) \leq$ $r_{n}\left(\varepsilon_{3}, K, d_{1}\right)$. Letting $n \rightarrow \infty \quad, \quad r_{d_{1}}\left(\varepsilon_{1}, K, f\right) \leq$ $r_{d_{2}}\left(\varepsilon_{2}, K, f\right) \leq r_{d_{1}}\left(\varepsilon_{3}, K, f\right)$. If $\quad \varepsilon_{1} \rightarrow 0$, then $\varepsilon_{2} \rightarrow 0$ and $\varepsilon_{3} \rightarrow 0$ so we have $h_{d_{1}}(f, K)=$ $h_{d_{2}}(f, K)$.

(ii) Clearly, $r_{n}\left(\varepsilon, K, f^{m}\right) \leq r_{m n}(\varepsilon, K, f)$; it follows that $\quad \frac{1}{n} \log r_{n}\left(\varepsilon, K, f^{m}\right) \leq \frac{m}{m n} \log r_{m n}(\varepsilon, K, f) \quad$ and therefore $h_{d}\left(f^{m}\right) \leq m \cdot h_{d}(f)$. Since $f \in \operatorname{UC}(X, d)$, given $\varepsilon>0$, there exist $\delta>0$ such that $d(x, y)<$ 
$\delta$ implies $\max _{0 \leq i \leq m-1} d\left(f^{i}(x), f^{i}(y)\right)<\varepsilon$. So an $(n, \delta)$-spanning set for $K$ with respect to $f^{m}$ is automatically an $(m n, \varepsilon)$-spanning set for $K$ with respect to $f$. Hence $r_{n}\left(\delta, K, f^{m}\right) \geq r_{m n}(\varepsilon, K, f)$, so $r\left(\delta, K, f^{m}\right) \geq m r(\varepsilon, K, f)$. Therefore $h_{d}\left(f^{m}, K\right) \geq$ $m \cdot h_{d}(f, K)$. So, $h_{d}\left(f^{m}\right) \geq m \cdot h_{d}(f)$.

If $X$ is compact and if $d$ and $d^{\prime}$ are equivalent metrics then they are uniformly equivalent. Also each continuous map $f: X \rightarrow X$ is uniformly continuous. Therefore if $X$ is compact metrizable space the entropy of $f$ does not depend on the metric chosen on $X$ (provided that metric induces the topology of $X)$.

Theorem 3.2. Let $(X, d)$ be a metric space and $f \in \mathrm{UC}(X, d)$. If $K \subseteq K_{1} \cup \cdots \cup K_{m}$ are all compact subsets of $X$, then

$$
h_{d}(f, K) \leq \max _{1 \leq i \leq m} h_{d}\left(f, K_{i}\right) .
$$

Proof. Clearly $s_{n}(\varepsilon, K) \leq s_{n}\left(\varepsilon, K_{1}\right)+\cdots+$ $s_{n}\left(\varepsilon, K_{m}\right)$. Fix $\varepsilon>0$. For each $n \in \mathbb{N}$, choose $K_{i(n, \varepsilon)}$ such that $s_{n}\left(\varepsilon, K_{i(n, \varepsilon)}\right)=\max _{1 \leq j \leq m} s_{n}\left(\varepsilon, K_{j}\right)$. Then $s_{n}(\varepsilon, K) \leq m \cdot s_{n}\left(\varepsilon, K_{i(n, \varepsilon)}\right)$ and so,

$$
\log s_{n}(\varepsilon, K) \leq \log m+\log s_{n}\left(\varepsilon, K_{i(n, \varepsilon)}\right) .
$$

Choose $n_{j} \rightarrow \infty$ such that

$$
\frac{1}{n_{j}} \log s_{n_{j}}(\varepsilon, K) \rightarrow \limsup _{n \rightarrow \infty} \frac{1}{n} \log s_{n}(\varepsilon, K)
$$

and so that $K_{i\left(n_{j}, \varepsilon\right)}$ does not depend on $j$ (i.e., $\left.K_{i\left(n_{j}, \varepsilon\right)}=K_{i(\varepsilon)} \forall j \quad\right) . \quad$ Therefore $\quad s(\varepsilon, K, f) \leq$ $s\left(\varepsilon, K_{i(\varepsilon)}, f\right)$. Choose $\varepsilon_{q} \rightarrow 0$ so that $K_{i\left(\varepsilon_{q}\right)}$ is constant $\left(=K_{i_{0}}\right.$, say). Henceh $h_{d}(f, K) \leq h_{d}\left(f, K_{i_{0}}\right) \leq$ $\max _{1 \leq i \leq m} h_{d}\left(f, K_{i}\right)$.

Corollary 3.1. Let $(X, d)$ be a metric space and $f \in \operatorname{UC}(X, d)$ and also let $\delta>0$. In order to compute $h_{d}(f)$, it is suffices to take the supremum of $h_{d}(f, K)$ over those compact subsets of diameter less than $\delta$.

Proof. Since $K$ is compact, it can be covered by a finite number of balls $B_{1}, \ldots, B_{m}$ of diameter $\frac{\delta}{2}$ and hence

$$
h_{d}(f, K) \leq \max _{1 \leq i \leq m} h_{d}\left(f, K \cap \operatorname{cl}\left(B_{i}\right)\right),
$$
so that $h_{d}(f)=\max _{1 \leq i \leq m} h_{d}\left(f, K \cap \operatorname{cl}\left(B_{i}\right)\right)$.

Corollary 3.2. If $X$ is a compact metrizable space and $d$ is any metric on $X$, then

$$
h(f)=h_{d}(f)=h_{d}(f, X)=h(f, X) .
$$

Proof. If $K$ is a compact subset of $X$, then $h_{d}(f, K) \leq h_{d}(f, X)$. It follows from Theorem 4(i) that $h_{d}(f)$ does not depend on $d$.

When $X$ is compact, Corollary 3.2 can be used to simplify the definition of $h(f)$. Take any metric $d$ giving the topology of $X$, then

$$
\begin{gathered}
h(f)=\lim _{\varepsilon \rightarrow 0} \limsup _{n \rightarrow \infty} \frac{1}{n} \log r_{n}(\varepsilon, X) \\
=\lim _{\varepsilon \rightarrow 0} \limsup _{n \rightarrow \infty} \frac{1}{n} \log s_{n}(\varepsilon, X) .
\end{gathered}
$$

These expressions have the following interpretation. Suppose we want to count the number of orbits of length $n$ (an orbit of length $n$ is a set $\left\{x, f(x), \ldots, f^{n-1}(x)\right\}$ but we can only measure to an error $\varepsilon$. Then both $r_{n}(\varepsilon, X)$ and $s_{n}(\varepsilon, X)$ can be interpreted as the number of orbits of length $n$ up to error $\varepsilon$. So as $\varepsilon \rightarrow 0, h(f)$ is a measurement of the growth rate in $n$ of the number of orbits of length $n$ up to error $\varepsilon$.

Other papers dealing with extensions of topological entropy to non-compact spaces are Hofer [8], Bowen [3], Canovas [4], Canovas\& Rodriguez [7], and Canovas\&Linero $[5,6]$.

Next we make a slight modification on the symbol of refinement. The properties of this modification also investigated below.

Definition 3.3. A cover $u$ is said to be a refinement of a cover $\mathcal{V}$, denoted as $U \prec \mathcal{V}$, if every member of $U$ is a subset of some member of $\mathcal{V}$. In other words, $\mathcal{U} \prec \mathcal{V}$ if $A \in \mathcal{U}$, there exists a $B \in \mathcal{V}$ such that $A \subseteq B$.

The following proposition is analogue results of Lemma 2.3.

Proposition 3.1. Let $\mathcal{U}, \mathcal{V}, \mathcal{U}^{\prime}$ and $\mathcal{V}^{\prime}$ be open covers of $X$. Then the following statements hold:

(i) $u<U^{\prime}, v \prec \mathcal{V}^{\prime} \Rightarrow U \vee v \prec \mathcal{U}^{\prime} \vee \mathcal{v}^{\prime}$. 
(ii) $\mathcal{U} \prec \mathcal{V} \Rightarrow N(\mathcal{U}) \geq N(\mathcal{V})$ and $H(\mathcal{U}) \geq H(\mathcal{V})$.

(iii) $\quad \mathcal{U} \prec \mathcal{v} \Rightarrow N(\mathcal{U} \vee \mathcal{V})=N(\mathcal{U})$ and $H(\mathcal{U} \vee$ $v=H U$.

(iv) $U<\mathcal{V} \Rightarrow f^{-1}(\mathcal{U}) \prec f^{-1}(\mathcal{V})$.

(v) $u \prec v \Rightarrow h(f, u) \geq h(f, v)$.

Proof. (i) Consider $A \cap B \in \mathcal{U} \vee \mathcal{V}$ where $A \in$ $\mathcal{U}$ and $B \in \mathcal{V}$. By hypothesis there exists $A^{\prime} \in \mathcal{U}^{\prime}$ and $B^{\prime} \in \mathcal{V}^{\prime}$ such that $A \subseteq A^{\prime}$ and $B \subseteq B^{\prime}$. Thus $A \cap B \subseteq A^{\prime} \cap B^{\prime}$ where $A^{\prime} \cap B^{\prime} \in \mathcal{U}^{\prime} \vee \mathcal{V}^{\prime}$.

(ii) Let $\left\{A_{1}, \ldots, A_{N(U)}\right\}$ be a subcover of $\mathcal{U}$ of minimal cardinality. Since $U \prec \mathcal{V}$, there exists a subcover $\left\{B_{1}, \ldots, B_{N}(u)\right\}$ of $\mathcal{V}$ such that $A_{i} \subseteq B_{i}$ for $i=1, \ldots, N(\mathcal{U})$. Therefore $N(\mathcal{U}) \geq N(\mathcal{V})$.

(iii) Obviously by (i), $\mathcal{U} \vee \mathcal{V} \prec \mathcal{U} \vee\{X\}=\mathcal{U}$ so that $N(\mathcal{U} \vee \mathcal{V}) \geq N(\mathcal{U})$ by (ii). On the other hand, $u<v$ and $u<\mathcal{U}$ implies $u<\mathcal{U} \vee \mathcal{U} \prec \mathcal{U} \vee v$. Thus $N(\mathcal{U}) \geq N(\mathcal{U} \vee \mathcal{V})$.

(iv) Let $A \in f^{-1}(\mathcal{U})$, then $f(A) \in \mathcal{U}$. Since $\mathcal{U} \prec \mathcal{V}$, there exists $B \in \mathcal{V}$ such that $f(A) \subseteq B$. This implies that $A \subseteq f^{-1}(f(A)) \subseteq f^{-1}(B) \in f^{-1}(\mathcal{V})$. Thus $f^{-1}(\mathcal{U}) \prec f^{-1}(\mathcal{V})$.

(v) By part (i) and (iv), it follows that $\mathcal{U} \vee f^{-1} \mathcal{U} \vee$ $\cdots \vee f^{-n+1} u \prec \mathcal{v} \vee f^{-1} \mathcal{v} \vee \cdots \vee f^{-n+1} \mathcal{v}$ for all $n \in \mathbb{N}$. Therefore by (ii)

$$
\begin{aligned}
H\left(\mathcal{U} \vee f^{-1} \mathcal{U} \vee\right. & \left.\cdots \vee f^{-n+1} \mathcal{u}\right) \\
& \geq H\left(\mathcal{V} \vee f^{-1} \mathcal{v} \vee \cdots \vee f^{-n+1} \mathcal{v}\right) .
\end{aligned}
$$

This yields the claim.

Theorem 3.3. For the topological entropy of iterates the following formula holds:

$$
h\left(f^{k}\right)=k h(f) \text { for all } k \in \mathbb{N} .
$$

Proof. The proof of first part is similar to the proof of Theorem 2.3. The proof for the second part is as follow. Since for any open cover $U, \vee_{i=0}^{n k-1} f^{-i} \mathcal{U}$ $\vee_{i=0}^{n-1}\left(f^{k}\right)^{-i} u$, we have

$$
h(f, \mathcal{U})=\lim _{n \rightarrow \infty} \frac{1}{n k} H\left(\mathrm{~V}_{i=0}^{n k-1} f^{-i}(\mathcal{U})\right)
$$

$$
\begin{gathered}
\geq \lim _{n \rightarrow \infty} \frac{1}{n k} H\left(\mathrm{~V}_{i=0}^{n-1}\left(f^{k}\right)^{-i} \mathcal{U}\right) \\
=\frac{1}{k} \lim _{n \rightarrow \infty} \frac{1}{n} H\left(\mathrm{~V}_{i=0}^{n-1}\left(f^{k}\right)^{-i} \mathcal{U}\right) \\
=\frac{1}{k} h\left(f^{k}, \mathcal{U}\right)
\end{gathered}
$$

by Proposition 3.1(ii). Thus $k h(f) \geq h\left(f^{k}\right)$.

\section{Acknowledgment}

The author gratefully acknowledges the Ministry of Education Malaysia, and University Malaysia Terengganu that this research was partially supported under the Fundamental Research Grant Scheme (FRGS) 59347.

\section{References}

[1] R.L. Adler, A.G. Konheim and M.H. McAndrew, Topological entropy, Trans. Amer. Math. Soc. 114(2) (1965) 309-319.

[2] R. Bowen, Entropy for group endomorphisms and homogeneous spaces, Trans. Amer. Math. Soc. 153 (1971) 401-414.

[3] R. Bowen, Topological entropy for noncompact sets, Trans. Amer. Math. Soc. 184 (1973)125-136.

[4] J.S. Cánovas, Topological entropy of continuous transitive maps on the real line, Dyn. Systems 24(4) (2009) 473-483.

[5] J.S. Canovas and A. Linero, On topological entropy of commuting interval maps, Nonlinear Analysis 51 (2002) 1159-1165.

[6] J.S. Canovas and A. Linero, On the dynamics of composition of commuting interval maps, J. Math. Anal. Appl. 305 (2005) 296-303.

[7] J.S. Cánovas and J.M. Rodríguez, Topological entropy of maps on the real line, Top. Appl.153 (2005) 735-746.

[8] J.E. Hofer, Topological entropy for noncompact spaces, Michigan Math. J. 21 (1974) 235-242.

[9] C. Robinson, Dynamical Systems: Stability, Symbolic Dynamics, and Chaos, 2nd Ed., Studies in Advanced Mathematics, CRC Press, Florida, 1999.

[10] P. Walters, An Introduction to Ergodic Theory, New York: Springer-Verlag, 1982. 